\title{
Correspondence
}

\section{Trials and tribulations of S49 orders}

The Mental Capacity Act 2005 (MCA 2005) is an Act of Parliament, applying to England and Wales, that provides a legal framework for acting and making decisions on behalf of adults who lack the capacity to make particular decisions for themselves. ${ }^{1}$ Under section 49 (pilot order) of the MCA 2005, launched in 2016, the Court of Protection can order reports from National Health Service (NHS) health bodies and local authorities when it is considering any question relating to someone who may lack capacity, and the report must deal with 'such matters as the court may direct'. ${ }^{2}$ This change has caused significant ethical challenges for psychiatrists.

With regard to professional implications, Section 49 reports require an opinion; according to British Medical Association (BMA) and General Medical Council (GMC) guidance, this falls under expert witness work. The recent Pool judgment is a reminder that the GMC is likely to consider that fitness to practice is impaired if a doctor acts outside what is considered their scope of work. ${ }^{3}$ The order is usually accompanied by an instruction letter containing legal precedents and a bundle sometimes containing conflicting assessments. Responding to such instructions require medico-legal training and experience in giving opinions to complex questions such as capacity to consent to sex, or consent to drink. We would argue that there is a blurring of boundaries between expert and professional witness. There is a need to clarify what legal safeguards are in place for the author of Section 49 reports, if their opinion is challenged, as it was in the Pool case.

In relation to patient care, the introduction of an automatic right to a medico-legal report, which was previously funded from elsewhere, has shifted the cost on to the NHS. Given that mental health services are still block funded; more work without additional funding leads to dilution of quality of care elsewhere in the system, affecting patient care. Lack of parity of esteem between physical and mental health funding makes this work an onerous burden. Increased workload without remuneration has an adverse effect on staff morale, influencing recruitment and retention within an already struggling NHS.

There is an urgent need to quantify the effects of these orders on services. The Royal College of Psychiatrists, working together with NHS England and the BMA, needs to define how medico-legal work can be safely done within existing resources. Moreover, the BMA, GMC, the College and NHS employers need to resolve the discrepancy that results from what is considered expert witness work by regulatory bodies being framed as normal NHS work by the Court of Protection. ${ }^{4}$ Legal safeguards need to be in place if NHS professionals become subject to legal challenge, e.g. from an aggrieved solicitor. Consideration needs to be given to a fresh legal challenge if it is evident that this pilot order is affecting patient care.

Ilyas Mirza and Mukesh Kripalani, Consultant Psychiatrist, Barnet Enfield and Haringey Mental Health NHS Trust; email: ilyasmirza@nhs.net
1 Mental Capacity Act 2005 Code of Practice. TSO, 2007 (https://www.gov. uk/government/publications/mental-capacity-act-code-of-practice).

2 Courts and Tribunals Judiciary. Court of Protection Transparency Pilot Case management S.49 pilots extension. Courts and Tribunal Judiciary, 2017 (https://www.judiciary.uk/publications/transparency-pilot-casemanagement-s-49-pilots-extension/).

3 Pool v General Medical Council [2014] EWHC 3791 (Admin).

4 General Medical Council (2013) Good Medical Practice; Acting as a witness in legal proceedings. GMC (https://www.gmc-uk.org/ethicalguidance/ethical-guidance-for-doctors/actingas-a-witness/acting-as-awitness-in-legal-proceedings).

doi:10.1192/bjb.2018.108

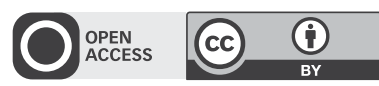

(c) The Authors 2019. This is an Open Access article, distributed under the terms of the Creative Commons Attribution licence (http://creativecommons.org/ licenses/by/4.0/), which permits unrestricted re-use, distribution, and reproduction in any medium, provided the original work is properly cited.

\section{The requirement for a general psychiatric assessment risks psychopathologising the experience of transgender people}

This paper and the service from which the statistics are drawn appear concerning on a number of levels. It appears unlikely that the fundamental assertion which underpins the statistics in this paper is accurate, namely that 'our case note review was able to capture all patients referred within a certain time period in this geographical area'.

The authors state that: 'All individuals who request treatment for gender dysphoria in Oxfordshire are referred to a single clinician (C.B.) for psychiatric assessment and subsequent referral to a specialist centre', and later, 'there is a single point of access in Oxfordshire for onward referral to specialist gender clinics'.

This referral pathway is not consistent with mainstream practice in other areas of England and is not supported by current protocols and guidelines representing best practice. The 2013 College Report Good Practice Guidelines for the Assessment and Treatment of Adults with Gender Dysphoria emphasises referral by or via a general practitioner, with no other gatekeeping requirement.

The requirement for a general psychiatric assessment is at best unnecessary and at worst risks psychopathologising the experience of transgender people who are presenting with gender dysphoria, an experience of discomfort or distress which is not psychiatric in nature.

There is local awareness of the unusual nature of the arrangement in Oxfordshire. The Oxford University LGBTQ+ society advises on its website: 'N.B. A lot of GPs will seek to refer trans customers to psychiatrists (in Oxford, this is usually Dr Chris Bass), but this is a completely unnecessary procedure. 
If you're seeking referral to a Gender Identity Clinic to receive hormone treatment or surgery, this will only lengthen the process. Psychiatric assessments are not required by Gender Identity Clinics, and your GP is qualified to make the referral'.

Given the high proportion of students who will have families of origin outside Oxfordshire and the high level of awareness within Oxford University that a psychiatric assessment is unnecessary, it appears likely that a significant number are bypassing the service.

Another factor not considered by the authors is the almost exponential rise in people presenting to gender identity services who are under the age of 17. Provision is via a centralised national service for young people. At 17, their care is transferred to an appropriate gender identity clinic. Transfers of care now comprise an increasing proportion of referrals to the Northern Region Gender Dysphoria Service. As this is the case nationally, such patients will also bypass Dr Bass and will not feature in the statistics presented.

Michael Shaw, Consultant in Gender Dysphoria, Northern Region Gender Dysphoria Service; email: NRGDS@ntw.nhs.uk

\section{doi:10.1192/bjb.2018.109}

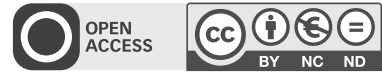

(c) The Author 2019. This is an Open Access article, distributed under the terms of the Creative Commons Attribution-NonCommercial-NoDerivatives licence (http://creativecommons.org/licenses/by-nc-nd/4.0/), which permits noncommercial re-use, distribution, and reproduction in any medium, provided the original work is unaltered and is properly cited. The written permission of Cambridge University Press must be obtained for commercial re-use or in order to create a derivative work.

\section{Contrast with referrals to Tavistock and Portman Gender Identity Disorder Service}

This article shows a modest increase in referrals year on year, approximately $18 \%$, with a majority of referrals received being for those assigned male at birth (AMAB). This is an interesting contrast with data from the Tavistock and Portman Gender Identity Disorder Service (GIDS) for children and adolescents.

Referrals to GIDS have increased from 97 in 2009/10 to 2016 in 2016/17. From 2014/15 to 2015/16, referrals increased by over $100 \%$ and from $2015 / 16$ to $2016 / 17$ they increased by $41 \%$. Ages at referral seen by the service ranged from a very few at 3 to 17 years old.

Also in contrast to this much larger increase in referral rates is a marked change in the proportion of those assigned female at birth (AFAB). Up until 2011 there were more referrals of those $A M A B$. Since then the number of those $A F A B$ referred has grown steadily, and in 2016/17 more than twice as many referrals were made for those $A F A B$ as those $A M A B$ (data available on GIDS website).

We need to be looking as a profession at these striking differences, and more research is required to determine the reasons for them. It may be that reducing stigma has led to higher referral rates, particularly among teenage girls but it could also be that the characteristics of those being referred are changing. This links with the finding that there seems to be a higher prevalence of autism spectrum conditions (ASC) in clinically referred, gender dysphoric adolescents than in the general adolescent population. Holt, Skagerberg and Dunsford (2014) found that $13.3 \%$ of referrals to the GIDS service in 2012 mentioned comorbid ASC (although this is likely to be an underestimate).

In this context, it is alarming that referral rates are increasing at a rate that services and research cannot keep up with. Both the American Academy of Pediatrics and the Australian Standards of Care and Treatment for Transgender and Gender Diverse Children and Adolescents appear to support both medical and surgical transition in adolescents. And yet long-term outcomes in this group are not known. We know that adults who have gender dysphoria and who transition report the dysphoria beginning in early childhood. We do not know yet know that those experiencing dysphoria in childhood will go onto experience dysphoria in adulthood. Indeed, we know that $80 \%$ of individuals referred to GIDS do not proceed to transition.

In the UK, we are fortunate to have a national service for children that follows the best available evidence, but there is an urgent need for both research and discussion. This is not always easy in a highly emotionally charged atmosphere.

Katherine Rae Clyde, Consultant in Older Persons' Mental Health, Royal College of Psychiatrists; email: katie.clyde@nhs.net

\section{doi:10.1192/bjb.2018.110}

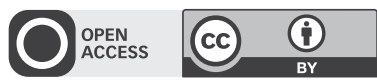

(C) The Author 2019. This is an Open Access article, distributed under the terms of the Creative Commons Attribution licence (http://creativecommons.org/ licenses/by/4.0/), which permits unrestricted re-use, distribution, and reproduction in any medium, provided the original work is properly cited.

Author reply: Our paper aimed to highlight the marked increase in referrals seen locally within recent years. We accept that, as Dr Shaw notes, some people, particularly those from the student population who also have families of origin outside Oxfordshire, may have been referred directly to gender identity clinics and so were not captured by the data. This would mean that our findings likely represent an underestimate of the total increase in numbers of people seeking assistance from specialist clinics, which makes the increase we did find even more striking.

We also appreciate Dr Shaw's points regarding the referral pathway in Oxfordshire and the need to minimise the barriers transgender people experience to accessing services. The clinical pathway has indeed been reviewed and altered since the period described in the study, with service users now being referred directly by general practitioners, generally to the specialist clinic in Northamptonshire.

The clinic did not assess people under the age of 17 , so we did not include data on this age group in our study, but it is certainly notable, as both Dr Shaw and Dr Clyde's letters highlight, that referrals to gender identity disorder services (GIDS) for children and adolescents have risen dramatically over the past five years. Dr Clyde in her letter draws attention to the high rates of referral to GIDS for children and adolescents since 2010, and in particular the increase in rates of 